\title{
Jost Function for Singular Potentials
}

\author{
S. A. Sofianos, S. A. Rakityansky, and S. E. Massen* \\ Physics Department, University of South Africa, P.O.Box 392, Pretoria 0003, South Africa
}

(September 17, 2018)

\begin{abstract}
An exact method for direct calculation of the Jost function and Jost solutions for a repulsive singular potential is presented. Within this method the Schrödinger equation is replaced by an equivalent system of linear first-order differential equations, which after complex rotation, can easily be solved numerically. The Jost function can be obtained to any desired accuracy for all complex momenta of physical interest, including the spectral points corresponding to bound and resonant states. The method can also be used in the complex angular-momentum plane to calculate the Regge trajectories. The effectiveness of the method is demonstrated using the Lennard-Jones $(12,6)$ potential. The spectral properties of the realistic inter-atomic ${ }^{4} \mathrm{He}^{-}{ }^{4} \mathrm{He}$ potentials HFDHE2 and HFD-B of Aziz and collaborators are also investigated.
\end{abstract}

PACS numbers: 03.65.Nk, 03.80.+r, 11.55.Hx, 34.20.Cf, 34.50.-s

\section{INTRODUCTION}

A new method for locating potential resonances and Regge trajectories, based on direct calculation of the Jost function in the complex $k$-plane, has recently been developed 1 i 1 . Within this method, the bound, resonant, and scattering states can be found by calculating the Jost solutions and the Jost function on the appropriate domain of the $k$-plane. The bound and resonant state energies, for example, can be found by locating the zeros of the Jost function on the positive imaginary axis and in the fourth quadrant respectively. At the same time, as a by-product of the Jost function calculation, one gets the physical wave function that has the correct asymptotic behavior.

The method, in the form developed in Refs. 佂, cannot be directly applied to potentials which are more singular than $1 / r^{2}$ at the origin. A significant number of practical problems, however, where the Jost function could be very useful, involves such potentials. For example, inter-atomic and inter-molecular forces at short distances are strongly repulsive due to the overlap of the electron clouds, and thus they are usually represented by repulsive singular potentials such as the Lennard-Jones $(12,6)$ one which has $\sim 1 / r^{12}$ behavior. It is, therefore, desirable to extend the Jost function method for potentials of this kind.

It is well-known that attractive singular potentials do not admit physically meaningful solutions with the usual boundary conditions [5]. All solutions of the Schrödinger equation with such potentials vanish at the origin and there is no apparent way to determine the arbitrary phase factor between them. In contrast, repulsive singular potentials do not pose any problem regarding mathematical uniqueness or physical interpretation. However, the integration of the Schrödinger equation as well as of the relevant equations for the Jost function, have inherent difficulties resulting from the fact that the singularity of the potential makes the $r=0$ an irregular singular point of the equation. In particular, the regular solution cannot be defined by universal boundary conditions independent of the potential.

This drawback, stemming from the extremely strong repulsion near the origin, can fortunately be tackled by the WKB approximation which provides the correct radial behavior of the wave function in the neighborhood of the point $r=0 \sqrt{6}$. Therefore, starting with the WKB boundary conditions, one can find the regular solution by integrating the Schrödinger equation from $r=0$ to some intermediate point $r=r_{\text {int }}$. Then the equations for the Jost function can be integrated, from $r_{\text {int }}$ outwards as for the nonsingular potential using the boundary conditions at $r=r_{\text {int }}$ which are expressed in terms of the regular solution and its first derivative.

The paper is organized as follows: In Sec. II our formalism is presented and is tested in Sec. III using an example known in the literature; in Sec. IV the method is applied to realistic interaction between helium atoms. Our conclusions are drawn in Sec. V.

*Permanent address: Department of Theoretical Physics, University of Thessaloniki, Thessaloniki, 54006, Greece 


\section{THEORY}

\section{A. Basic equations and definitions}

There are three different types of physical problems associated with the Schrödinger equation $\left(2 \mu / \hbar^{2}=1\right)$,

$$
\left[\partial_{r}^{2}+k^{2}-\ell(\ell+1) / r^{2}\right] u_{\ell}(k, r)=V(r) u_{\ell}(k, r),
$$

namely, bound, scattering, and resonant state problems. They differ in the boundary conditions imposed on the wave function at large distances. Alternatively, a solution can be prescribed by the boundary conditions at the origin. In the case of regular potentials obeying the condition

$$
\lim _{r \rightarrow 0} r^{2} V(r)=0
$$

the solution $\phi_{\ell}(k, r)$ which vanishes near $r=0$ exactly like the Riccati-Bessel function,

$$
\lim _{r \rightarrow 0} \phi_{\ell}(k, r) / j_{\ell}(k r)=1 \text {, }
$$

is called the regular solution. Since all physical solutions are regular at the origin, they differ from $\phi_{\ell}(k, r)$ only by a normalization constant. Therefore, if the function $\phi_{\ell}(k, r)$ can be calculated at all real and complex momenta $k$, one can have, in principle, all solutions of physical interest in a most general form. For example, the calculation of the scattering solutions on the real $k$-axis is simply a matter of finding the proper normalization for $\phi_{\ell}(k, r)$ because the regular solution has the correct behavior at large $r$ for any $k>0$. In contrast, to find the bound and resonant states where the $k$ is complex, one must ensure that the function $\phi_{\ell}(k, r)$ has the proper physical asymptotic behavior which exist only at certain points on the $k$-plane. These spectral points can be found by many different ways, but perhaps the most convenient way to find them is by locating the zeros of the Jost function.

For any complex $k$, the regular solution at large distances can be expressed as a linear combination of the RiccatiHankel functions $h_{\ell}^{( \pm)}(k r)$,

$$
\phi_{\ell}(k, r) \underset{r \rightarrow \infty}{\longrightarrow} \frac{1}{2}\left[h_{\ell}^{(+)}(k r) f_{\ell}^{*}\left(k^{*}\right)+h_{\ell}^{(-)}(k r) f_{\ell}(k)\right]
$$

where the $r$-independent but momentum-dependent coefficient $f_{\ell}(k)$ is the Jost function. From the asymptotic form (4) it is clear that the zeros of $f_{\ell}(k)$ on the positive imaginary axis of the complex $k$-plane correspond to bound states while those in the fourth quadrant to resonances.

In order to find $f_{\ell}(k)$, we look for the regular solution on the whole interval $[0, \infty)$ in the form

$$
\phi_{\ell}(k, r)=\frac{1}{2}\left[h_{\ell}^{(+)}(k r) F_{\ell}^{(+)}(k, r)+h_{\ell}^{(-)}(k r) F_{\ell}^{(-)}(k, r)\right],
$$

where the new unknown functions $F_{\ell}^{( \pm)}(k, r)$ are subjected to the additional condition

$$
h_{\ell}^{(+)}(k r) \partial_{r} F_{\ell}^{(+)}(k, r)+h_{\ell}^{(-)}(k r) \partial_{r} F_{\ell}^{(-)}(k, r)=0 \text {. }
$$

Eq. (11) is then transformed into an equivalent system of first order equations

$$
\partial_{r} F_{\ell}^{( \pm)}(k, r)= \pm \frac{h_{\ell}^{(\mp)}(k r)}{2 i k} V(r)\left[h_{\ell}^{(+)}(k r) F_{\ell}^{(+)}(k, r)+h_{\ell}^{(-)}(k r) F_{\ell}^{(-)}(k, r)\right]
$$

In Refs. 11,4] it was shown that at large distances $F_{\ell}^{(-)}(k, r)$ coincides with the Jost function,

$$
\lim _{r \rightarrow \infty} F_{\ell}^{(-)}(k, r)=f_{\ell}(k)
$$

but this limit only exists when

$$
\operatorname{Im} k r \geq 0 \text {. }
$$

If $r$ is real, the condition (9) is only satisfied for bound and scattering states but not for resonances. To calculate $f_{\ell}(k)$ we, therefore, make a complex rotation of the coordinate in Eqs. (7), in the first quadrant

$$
r=x \exp (i \theta), \quad x \geq 0, \quad 0 \leq \theta<\frac{\pi}{2},
$$

with a sufficiently large $\theta$ (see Refs. 1 1 - for more details). Such a rotation is only possible if the potential is an analytic function of $r$ and tends to zero when $x \rightarrow \infty$ for the chosen angle $\theta$. 


\section{B. Boundary conditions}

In the case of regular potentials the boundary conditions for Eqs. (7) are very simple,

$$
F_{\ell}^{( \pm)}(k, 0)=1
$$

They follow immediately from (3), (5), and the fact that

$$
\frac{1}{2}\left[h_{\ell}^{(+)}(k r)+h_{\ell}^{(-)}(k r)\right]=j_{\ell}(k r) .
$$

Going over to singular potentials, Eq. (3) does not hold anymore. Due to the extremely strong repulsion, the regular solution vanishes much faster than $j_{\ell}(k r)$ when $r \rightarrow 0$. In fact, it vanishes exponentially [7] and therefore the conditions (11) must be modified accordingly. In order to find the exact behavior of the regular solution near the origin we apply the familiar semi-classical WKB method. Though the strong repulsion makes things rather complicated, it has the advantage that the criterion of the applicability of the WKB approximation is satisfied when $r \rightarrow 0$. Indeed, the WKB method works well when the local wavelength $\lambda$ varies slowly, i.e.

$$
|\mathrm{d} \lambda / \mathrm{d} r| \ll 1 .
$$

It can be shown [5] that this derivative is given by

$$
|\mathrm{d} \lambda / \mathrm{d} r|=\frac{1}{2}\left|\frac{\mathrm{d} V(r)}{\mathrm{d} r}\left[k^{2}-V(r)\right]^{-3 / 2}\right| .
$$

Assuming that $V(r)$ approaches its singularity near $r=0$ monotonically, we can find an $r_{\min }$ that for all $r<r_{\min }$ the momentum in (13) is negligible, i.e. we may write

$$
|\mathrm{d} \lambda / \mathrm{d} r| \underset{r \rightarrow 0}{\longrightarrow} \frac{1}{2}\left|\frac{\mathrm{d} V(r)}{\mathrm{d} r}[V(r)]^{-3 / 2}\right|
$$

When $r \rightarrow 0$, the right hand side of Eq. (14) for usual singular potentials tends to zero. For example, if

$$
V(r) \underset{r \rightarrow 0}{\longrightarrow} g / r^{n},
$$

the condition (12) is always satisfied for $n>2$,

$$
|\mathrm{d} \lambda / \mathrm{d} r| \underset{r \rightarrow 0}{\longrightarrow} \frac{n r^{\frac{1}{2} n-1}}{2 \sqrt{g}} \longrightarrow 0, \quad \text { if } n>2 .
$$

Therefore, assuming that the necessary condition (12) is fulfilled and choosing a small enough $r_{\text {min, }}$ we can express the regular solution on the interval $\left[0, r_{\mathrm{min}}\right]$ using the WKB approximation (see, for example, Ref. [8]), viz.

$$
\phi_{\ell}(k, r)=\frac{1}{\sqrt{p(r)}} \exp \left[i \int_{r}^{a} p(\rho) \mathrm{d} \rho\right], \quad r \in\left[0, r_{\min }\right],
$$

where the classical momentum $p(r)$ is defined by

$$
p(r) \equiv \sqrt{k^{2}-V(r)-\left(\ell+\frac{1}{2}\right)^{2} / r^{2}}
$$

and the upper limit $a$ in the integral is an arbitrary value $a>r_{\min }$. Usually $a$ is taken to be the inner turning point [8], but it is obvious from Eq. (15) that an additional integration from $a$ to the turning point can only change the overall normalization of the solution which is not our concern at the moment. Thus, Eq. (15) together with the derivative

$$
\partial_{r} \phi_{\ell}(k, r)=\left\{\frac{\frac{\mathrm{d} V(r)}{\mathrm{d} r}-2\left(\ell+\frac{1}{2}\right)^{2} r^{-3}}{4[p(r)]^{5 / 2}}-i \sqrt{p(r)}\right\} \exp \left[i \int_{r}^{a} p(\rho) \mathrm{d} \rho\right], \quad r \in\left(0, r_{\min }\right]
$$


can be used as boundary conditions for the regular solution of the Schrödinger equation at any point in the interval $\left(0, r_{\min }\right]$. To obtain the corresponding boundary conditions for the functions $F_{\ell}^{( \pm)}(k, r)$, we need to express them in terms of $\phi_{\ell}(k, r)$ and $\partial_{r} \phi_{\ell}(k, r)$. For this we can use Eq. (5) together with relation

$$
\partial_{r} \phi_{\ell}(k, r)=\frac{1}{2}\left[F_{\ell}^{(+)}(k, r) \partial_{r} h_{\ell}^{(+)}(k r)+F_{\ell}^{(-)}(k, r) \partial_{r} h_{\ell}^{(-)}(k r)\right]
$$

which follows from (6). From (5) and (18) we find that

$$
F_{\ell}^{( \pm)}(k, r)= \pm \frac{i}{k}\left[\phi_{\ell}(k, r) \partial_{r} h_{\ell}^{(\mp)}(k r)-h_{\ell}^{(\mp)}(k r) \partial_{r} \phi_{\ell}(k, r)\right]
$$

which is valid for any $r \in[0, \infty)$. Therefore Eqs. (19) taken at some point $r<r_{\min }$ with $\phi_{\ell}(k, r)$ and $\partial_{r} \phi_{\ell}(k, r)$ given by (15) and (17), provide us the boundary conditions, required in Eqs. (7), for singular potentials. It can easily be checked (by using $j_{\ell}(k r)$ for the regular solution near $r=0$ ) that Eq. (19) gives the correct boundary conditions for regular potentials as well,

Alternatively to impose the boundary conditions on the functions $F_{\ell}^{( \pm)}(k, r)$ near the origin, one can simply solve the Schrödinger equation from a small $r$ up to some intermediate point $b$ where, using $(19)$, the $F_{\ell}^{( \pm)}(k, b)$ can be obtained and propagated further on by integrating equations (7).

\section{Integration path}

The use of more complicated boundary conditions at $r=0$ does not change the condition (9) for the existence of the limit (8). Indeed, in deriving this condition we used only the behavior of the potential and the Riccati-Hankel functions at large distances [1] t]. Therefore, the Jost function for a singular potential can also be calculated by evaluating the function $F_{\ell}^{(-)}(k, r)$ at a large $r$. When we are dealing with resonances, i.e. working in the fourth quadrant of the $k$-plane, we need to integrate Eqs. (7) along the turned ray (10).

As can be seen from the WKB boundary conditions 15$)$, the use of a complex $r$ near the origin, makes $\phi_{\ell}(k, r)$ oscillatory from the outset. Although this does not formally cause any problem, in numerical calculations such oscillations may reduce the accuracy. To avoid this we solve Eqs. (7) from a small $r_{\text {min }}$ to some intermediate point $b$ along the real axis and then perform the complex rotation,

$$
r=b+x \exp (i \theta), \quad x \in[0, \infty), \quad 0 \leq \theta<\frac{\pi}{2}
$$

as is shown in Fig. 11. Therefore, on the interval $\left[r_{\min }, b\right]$ we can use Eqs. (7) as they are, while beyond the point $r=b$ these equations are transformed to

$$
\begin{aligned}
\partial_{x} F_{\ell}^{( \pm)}\left(k, b+x e^{i \theta}\right)= & \pm \frac{e^{i \theta} h_{\ell}^{(\mp)}\left(k b+k x e^{i \theta}\right)}{2 i k} V\left(b+x e^{i \theta}\right)\left[h_{\ell}^{(+)}\left(k b+k x e^{i \theta}\right) F_{\ell}^{(+)}\left(k, b+x e^{i \theta}\right)\right. \\
& \left.+h_{\ell}^{(-)}\left(k b+k x e^{i \theta}\right) F_{\ell}^{(-)}\left(k, b+x e^{i \theta}\right)\right]
\end{aligned}
$$

Though the complex transformation (20) is different from (10), the proof of the existence of the limit (8) given in the Appendix A.2 of Ref. [4] remains applicable here. Indeed, that proof was based on the fact that for Im $k r>0$ the Riccati-Hankel function $h_{\ell}^{(+)}(k r)$ decays exponentially at large $|r|$, and thus the derivative $\partial_{r} F^{(-)}(k, r)$ vanishes there and the function $F^{(-)}(k, r)$ becomes a constant. Under the transformation (20) the asymptotic behavior of the Riccati-Hankel function,

$$
h_{\ell}^{(+)}(k r) \underset{r \rightarrow \infty}{\longrightarrow}-i \exp [i(k r-\ell \pi / 2)]
$$

has only an additional $r$-independent phase factor $\exp (i k b)$ which does not affect the proof.

From the above, it is clear that we can identify the Jost function $f_{\ell}(k)$ as the value of $F_{\ell}^{(-)}\left(k, b+x e^{i \theta}\right)$ at a sufficiently large $x$ beyond which this function is practically constant. In the bound and scattering state domain, 
where $\operatorname{Im} k \geq 0$, one can choose any rotation angle $\theta$ allowed by the potential, including $\theta=0$. In the resonance domain, however, where

$$
k=|k| \exp (-i \varphi), \quad \varphi>0,
$$

the rotation angle $\theta$ must be greater or equal to $\varphi$. If the condition $\theta \geq \varphi$ is fulfilled, the value of the limit (8) does not depend on the choice of $\theta$. This provides us with a reliable way to check the stability and accuracy of the calculations by comparing the results for $f_{\ell}(k)$ obtained with two different values of $\theta$.

From Eq. (22) it is clear that the angular momentum appears only in the phase factor of the asymptotic behavior of the Riccati-Hankel functions and hence of the regular solution. Therefore, the use of any complex $\ell$ cannot change the domain of the $k$-plane where the limit (8) exists. This means that the Jost function can be calculated, for any complex angular momentum, using the same equations. Moreover, when looking for the Regge poles in the $\ell-$ plane, the complex rotation is not necessary because these poles correspond to real energies. Locating Regge poles as zeros of the Jost function in the complex $\ell$-plane is easier than by calculating them via the $S$-matrix using three integration paths (in the $r$-plane) as suggested in Ref. 9.

\section{Jost solutions}

By storing the values of $F_{\ell}^{( \pm)}(k, r)$ on the integration grid one can also obtain the regular solution in the form (5) on the interval $\left[r_{\min }, r_{\max }\right]$. It is noted that the use of the Riccati-Hankel functions in (5) guarantees the correct (in fact exact) asymptotic behavior of the wave function.

The regular solution thus obtained consists of two terms:

$$
\begin{aligned}
& \frac{1}{2} h_{\ell}^{(+)}(k r) F_{\ell}^{(+)}(k, r) \underset{r \rightarrow \infty}{\longrightarrow}-\frac{i}{2} \exp [+i(k r-\ell \pi / 2)] f_{\ell}^{*}\left(k^{*}\right), \\
& \frac{1}{2} h_{\ell}^{(-)}(k r) F_{\ell}^{(-)}(k, r) \underset{r \rightarrow \infty}{\longrightarrow}+\frac{i}{2} \exp [-i(k r-\ell \pi / 2)] f_{\ell}(k) .
\end{aligned}
$$

Asymptotically they behave like $\sim \mathrm{e}^{ \pm i k r}$ and thus at long distances they are proportional to the commonly used Jost solutions $\mathrm{f}_{\ell}^{( \pm)}(k, r)$ for which

$$
\mathrm{f}_{\ell}^{( \pm)}(k, r) \underset{r \rightarrow \infty}{\longrightarrow} h_{\ell}^{( \pm)}(k r) .
$$

In practice, the Jost solutions can be calculated, via (5), by integrating Eqs. (7) inwards from a sufficiently large $r_{\max }$ with the boundary conditions

$$
\begin{aligned}
& {\left[\begin{array}{l}
F_{\ell}^{(+)}\left(k, r_{\max }\right) \\
F_{\ell}^{(-)}\left(k, r_{\max }\right)
\end{array}\right]=\left[\begin{array}{l}
2 \\
0
\end{array}\right], \quad \text { for } \mathrm{f}_{\ell}^{(+)}(k, r),} \\
& {\left[\begin{array}{l}
F_{\ell}^{(+)}\left(k, r_{\max }\right) \\
F_{\ell}^{(-)}\left(k, r_{\max }\right)
\end{array}\right]=\left[\begin{array}{l}
0 \\
2
\end{array}\right], \quad \text { for } \mathrm{f}_{\ell}^{(-)}(k, r),}
\end{aligned}
$$

which obviously comply with the definition (23). The advantage of such an approach is that at large $r$ all the oscillations of $\mathrm{f}_{\ell}^{( \pm)}(k, r)$ are described exactly by the Riccati-Hankel functions while the functions $F_{\ell}^{( \pm)}(k, r)$ are smooth.

\section{LENNARD-JONES POTENTIAL}

In order to evaluate the accuracy and efficiency of our method we apply it to the Lennard-Jones potential

$$
V(r)=D\left[\left(\frac{d}{r}\right)^{12}-2\left(\frac{d}{r}\right)^{6}\right]
$$


which is well-known in atomic and molecular physics. Combined with a rotational barrier, this potential supports narrow as well as broad resonant states (see, for example, Ref. [8]). To locate them, any method employed must be pushed to the extreme, thus exhibiting its advantages and drawbacks.

To be able to compare our results with other calculations, we chose the parameters in (24) to be the same as those used in Refs. [8.10], namely, $d=3.56$ Åand with $D$ varying from $5 \mathrm{~cm}^{-1}$ to $60 \mathrm{~cm}^{-1}$. The choice $D=60 \mathrm{~cm}^{-1}$ together with the conversion factor $\hbar^{2} / 2 \mu=8.7802375 \mathrm{~cm}^{-1} \AA^{2}$ (which was used for all values of $D$ ) approximately represents the interaction between the Ar atom and the $\mathrm{H}_{2}$ molecule [8].

In Tables 1 and II the energies and widths of the first resonant states in the partial wave $\ell=8$ are presented for different values of $D$. The results obtained with three other methods described in Refs. [8,10] are also given. The digits shown there are stable under changes of the rotation angle and thus they indicate the accuracy achieved. The third column of these tables, contains the results obtained in Ref. [8] using a Complex Rotation (CR) method which in some aspects is similar to ours. The authors of that reference perform the rotation directly in the Schrödinger equation and integrate it from $r=0$ outwards and from a large $r_{\max }$ inwards. At the origin they use the WKB boundary conditions and at $r_{\max }$ they start from the Siegert spherical wave. In other words, the wave function is calculated using physical boundary conditions. In such an approach a resonance corresponds to a complex energy which matches the inward and outward integration. As indicated in Ref. [8], this method fails for broad resonances due to instability in the outward integration. In the fourth column the results obtained in Ref. [8] using the Quantum Time Delay (QTD) method are cited. This method is expected to be reliable for narrow resonances but its applicability to broad states is questionable. Finally, in the last column of Table I and II we give the results obtained in Ref. [10] using the Finite Range Scattering Wave (FRSW) method. The main idea of this method is based on the fact that while the scattering wave function cannot be expanded properly by a finite number of square integrable functions on an infinite range, it is possible to do so for a finite range.

The test calculations show that our method works well, especially for narrow resonances. Broad resonances can also be located. In contrast to the CR-method of Ref. [8], which was unstable for broad resonances corresponding to $D<35 \mathrm{~cm}^{-1}$, we succeeded even in the case of $D=5 \mathrm{~cm}^{-1}$ which generates an extremely broad state (its width is greater than the resonance energy by a factor of 2). Our results for small values of $D$, reproduce well the curve depicted in Fig. 3 of Ref. [8] which was produced semi-classically. The greater stability of the Jost function method as compared to the CR-method of Ref. [8] can be attributed to the use of the ansatz (5) for the regular solution. The Riccati-Hankel functions, explicitly extracted there, describe correctly all oscillations at large distances with the remaining functions $F_{\ell}^{( \pm)}$being smooth. Another reason for this stability is the use of the deformed integration path shown on Fig. 1, which enables us to avoid fast oscillations at short distances.

\section{AZIZ POTENTIALS}

The model potential considered in the previous section, though of typical form for inter-molecular interactions, does not describe any real physical system. To give a more practical example, we apply our formalism to study the interaction between two ${ }^{4} \mathrm{He}$ atoms. This interaction is of interest in the Bose-Einstein condensation and super-fluidity of helium at extremely low temperatures. It is known that two helium atoms form a dimmer molecule with binding energy of $\sim 1 \mathrm{mK}$, but, to the best of our knowledge, the possibility of forming dimmer resonances has not been investigated yet.

The search for a realistic ${ }^{4} \mathrm{He}^{-}{ }^{4} \mathrm{He}$ potential is a long-standing problem in molecular physics. The earliest successful potential of the Lennard-Jones(12-6) form was fitted just to reproduce the second virial coefficient. Later on some other characteristics of helium gas, such as viscosity, were included into the fitting (for a more detail review see Refs. [11,12]). Nowadays, the potentials suggested by Aziz and co-workers are considered as be realistic. Therefore, in this section, we apply our method using two versions of these potentials, namely, the HFDHE2 [11] and the HFD-B [12] potentials. They can both be described using the same analytical form

$$
\begin{aligned}
V(r) & =\varepsilon\left[A \exp \left(-\alpha \zeta-\beta \zeta^{2}\right)-\left(\frac{C_{6}}{\zeta^{6}}+\frac{C_{8}}{\zeta^{8}}+\frac{C_{10}}{\zeta^{10}}\right) F(\zeta)\right], \\
F(\zeta) & = \begin{cases}\exp \left[-(B / \zeta-1)^{2}\right] & , \text { if } \zeta \leq B, \\
1 & , \text { if } \zeta>B, \\
\zeta & =r / r_{m},\end{cases}
\end{aligned}
$$


but with different choices of the parameters (see Table [II]). The only principal difference in the functional form between them is the absence of the Gaussian term $(\beta=0)$ in the HFDHE2 potential.

Formally, the HFDHE2 and HFD-B are regular potentials since the presence of the cut-off function $F(\zeta)$ in $(25)$ makes them finite at $r=0$,

$$
V(r) \underset{r \rightarrow 0}{\longrightarrow} \varepsilon A .
$$

However, the product $\varepsilon A$ is very large $\left(\sim 10^{6}\right)$ as compared with the values of the potential in the attractive region. This causes numerical instabilities when one tries to solve the Schrödinger equation using methods designed for regular potentials. To avoid this difficulty, we notice that like in the case of singular potentials the fast growth of the repulsion near the origin allows the use of the WKB boundary conditions near $r=0$. Indeed, the derivative of the potential in the vicinity of this point,

$$
\frac{\mathrm{d} V}{\mathrm{~d} r} \underset{r \rightarrow 0}{\longrightarrow}-\frac{\alpha \varepsilon A}{r_{m}}
$$

is of the same order of magnitude as $V$, which makes the derivative of the local wavelength (14) very small because of the large $A$,

$$
|\mathrm{d} \lambda / \mathrm{d} r| \underset{r \rightarrow 0}{\longrightarrow} \frac{\alpha}{2 r_{m} \sqrt{\frac{2 \mu}{\hbar^{2}} \varepsilon A}},
$$

where the conversion factor $\hbar^{2} / 2 \mu=12.12 \mathrm{~K} \AA^{2}$, corresponding to the choice of the units in Table III, should be used. With the parameters given, formula (28) gives 0.003 and 0.004 for the potentials HFDHE2 and HFD-B respectively. These values of $|\mathrm{d} \lambda / \mathrm{d} r|$ are small enough to comply with (12) and allow the use of WKB boundary conditions. We can, therefore, apply the method described in the preceding sections, to the potentials HFDHE2 and HFD-B as if they were singular potentials.

To begin with, we tested the ability of our method to deal with this kind of potentials by calculating the dimmer binding energy. The results of these calculations are given in Table IV where, for comparison, we also cite the binding energies obtained in several earlier works. It is seen that the potentials HFDHE2 and HFD-B support a dimmer bound state at energies which differ by a factor of 2 .

A question then arises whether these potentials generate also quite different distribution of resonances which would result in different on and off the energy shell characteristics of the scattering amplitude. To study this we located several zeros of the Jost function in the momentum as well as in the $\ell$-plane (Regge poles) for both potentials. Due to the absence of a potential barrier there are no resonances in the $S$-wave (at least with a reasonably small width). They appear, however, at higher partial waves, starting from $\ell=1$. The energies and widths of several such resonances are given in Table $\mathrm{V}$. They are the lowest resonant states in each partial wave as they belong to the same Regge trajectory which starts from the ground state. The trajectories for the potentials HFDHE2 and HFD-B are practically indistinguishable and are shown in Fig. 2 by a single curve. Few points of this curve which correspond to resonances, are also given in Table VI. It is seen that, to all practical purposes, the position of the Regge poles are the same.

As can be seen in Table $\mathrm{V}$, in each partial wave the potential generates a broad resonance which covers the whole low-energy region. This, together with the fact that the bound state pole of the amplitude is very close to $k=0$, implies that the cross-section at energies $\sim 10^{\circ} \mathrm{K}\left(\sim 10^{-3} \mathrm{eV}\right)$ must be quite large.

\section{CONCLUSIONS}

We presented an exact method for calculating the Jost solutions and the Jost function for singular potentials, for real or complex momenta of physical interest. We demonstrated in the examples considered, the suggested method is sufficiently stable and effective not only in the case of true singular potentials but also when a potential has strong, though finite, repulsion at short distances. 
The method is based on simple differential equations of the first order, which can be easily solved numerically. Thus, the spectrum generated by any given potential can be thoroughly investigated. At the same time, physical wave function can be obtained having the correct asymptotic behavior. When the potential has a Coulomb tail one can simply replace the Riccati-Hankel functions in the relevant equations by their Coulomb analogous, $H_{\ell}^{( \pm)}(\eta, k r) \equiv F_{\ell}(\eta, k r) \mp i G_{\ell}(\eta, k r)$ 2. In the case of a non-central potential the Jost function as well as the differential equations assume a matrix form with somewhat more complicated, but still tractable boundary conditions at $r=0$ 沟.

The method is also applicable when the angular momentum is complex. This enables us to locate Regge trajectories as well. This could be useful, for example, in molecular scattering problems where the partial wave series in many cases converges slowly [17]. This slow convergence can be overcome by allowing the angular momentum to become complex valued which allows the use of the Watson transformation. However, such a procedure requires the knowledge of the positions of the Regge poles.

\section{ACKNOWLEDGEMENTS}

Financial support from the University of South Africa, the Foundation for Research Development (FRD) of South Africa, and the Joint Institute for Nuclear Research (JINR), Dubna, is greatly appreciated. 
[1] S. A Rakityansky, S. A. Sofianos, and K. Amos, Nuovo Cimento 111 B, 363 (1996).

[2] S. A. Sofianos and S. A. Rakityansky, J. Phys. A: Math. Gen. 30, 3725 (1997).

[3] S. A. Sofianos, S. A. Rakityansky, and G. P. Vermaak, J. Phys. G: Nucl. Part. Phys. 23, 1619 (1997).

[4] S. A Rakityansky and S. A. Sofianos, J. Phys. A: Math. Gen. 31, 5149 (1998).

[5] W. M. Frank and D. J. Land, Rev. Mod. Phys. 43, 36 (1971).

[6] L. D. Landau and E. M. Lifshitz, Quantum Mechanics (Pergamon, Oxford, 1965).

[7] R. G. Newton, Scattering Theory of Waves and Particles, 2nd ed. (Springer, New York, 1982).

[8] J. N. L. Connor and A. D. Smith, J. Chem. Phys., 78, 6161 (1983).

[9] C. V. Sukumar and J. N. Bardsley, J. Phys. B 8, 568 (1975).

[10] H. W. Jang and J. C. Light, J. Chem. Phys. 99, 1057 (1993).

[11] R. A. Aziz, V. P. Nain, J. S. Carley, W. L. Taylor, G. T. McConville, J. Chem. Phys., 70, 4330 (1979).

[12] R. A. Aziz et al., Mol. Phys., 61, 1487 (1987).

[13] E. A. Kolganova, A. K. Motovilov, and S. A. Sofianos, J. Phys. B: At. Mol. Opt. Phys., 31, 1279 (1998).

[14] T. Cornelius and W. Glöckle, J. Chem. Phys., 85, 3906 (1986).

[15] S. Nakaichi-Maeda and T. K. Lim, Phys. Rev. A, 28, 692 (1983).

[16] Y. H. Uang and W. C. Stwalley, J. Chem. Phys. 76, 5069 (1982).

[17] J. N. L. Connor, J. Chem. Soc. Faraday Trans. 86, 1627 (1990). 


\begin{tabular}{|c|c|c|c|c|}
\hline $\begin{array}{c}\text { Ref. } \\
D\left(\mathrm{~cm}^{-1}\right)\end{array}$ & $\begin{array}{l}\text { This work } \\
E_{\text {res }}\left(\mathrm{cm}^{-1}\right)\end{array}$ & $\begin{array}{l}\text { CR } \\
E_{\mathrm{res}}\left(\mathrm{cm}^{-1}\right)\end{array}$ & $\begin{array}{l}\text { QTD } \\
E_{\mathrm{res}}\left(\mathrm{cm}^{-1}\right)\end{array}$ & $\begin{array}{l}\text { FRSW 10 10 } \\
E_{\text {res }}\left(\mathrm{cm}^{-1}\right.\end{array}$ \\
\hline 5 & 30 & & & \\
\hline 10 & 27 & & & \\
\hline 15 & 25.5 & & & \\
\hline 20 & 24.5 & & & \\
\hline 25 & 22.90 & & & \\
\hline 30 & 21.193 & & & \\
\hline 35 & 19.450 & 19.449 & 19.370 & \\
\hline 40 & 17.6478 & 17.647 & 17.619 & 17.617 \\
\hline 45 & 15.7768 & 15.777 & 15.769 & 15.769 \\
\hline 50 & 13.81980 & 13.820 & 13.819 & 13.818 \\
\hline 55 & 11.744242 & 11.744 & 11.744 & 11.743 \\
\hline 60 & 9.4943275 & 9.494 & 9.494 & 9.4934 \\
\hline
\end{tabular}

TABLE I. Energies of the lowest resonances, in the $\ell=8$ partial wave, for the Lennard-Jones potential with different $D$.

\begin{tabular}{|c|c|c|c|c|}
\hline \begin{tabular}{|c|c|} 
Ref. \\
$D\left(\mathrm{~cm}^{-1}\right)$ \\
\end{tabular} & $\begin{array}{l}\text { This work } \\
\Gamma_{\text {res }}\left(\mathrm{cm}^{-1}\right)\end{array}$ & $\begin{array}{l}\text { CR } \\
\Gamma_{\text {res }}\left(\mathrm{cm}^{-1}\right)\end{array}$ & $\begin{array}{l}\text { QTD } \\
\Gamma_{\text {res }}\left(\mathrm{cm}^{-1}\right)\end{array}$ & $\begin{array}{l}\text { FRSW } \llbracket 10 \\
\Gamma_{\text {res }}\left(\mathrm{cm}^{-1}\right)\end{array}$ \\
\hline 5 & 60 & & & \\
\hline 10 & 42 & & & \\
\hline 15 & 29.4 & & & \\
\hline 20 & 24.8 & & & \\
\hline 25 & 18.63 & & & \\
\hline 30 & 13.70 & & & \\
\hline 35 & 9.724 & 9.727 & 10.228 & \\
\hline 40 & 6.533 & 6.536 & 6.604 & 6.603 \\
\hline 45 & 4.039 & 4.039 & 3.992 & 3.990 \\
\hline 50 & 2.1833 & 2.183 & 2.143 & 2.142 \\
\hline 55 & 0.93915 & 0.939 & 0.926 & 0.926 \\
\hline 60 & 0.264474 & 0.264 & 0.263 & 0.263 \\
\hline
\end{tabular}

TABLE II. Widths of the lowest resonances, in the $\ell=8$ partial wave, for the Lennard-Jones potential with different $D$.

\begin{tabular}{|c|l|l|}
\hline \hline parameter & HFDHE2 & HFD-B \\
\hline$\varepsilon(\mathrm{K})$ & 10.8 & 10.948 \\
$r_{m}(\AA)$ & 2.9673 & 2.963 \\
$A$ & 544850.4 & 184431.01 \\
$\alpha$ & 13.353384 & 10.43329537 \\
$\beta$ & 0 & 2.27965105 \\
$C_{6}$ & 1.3732412 & 1.36745214 \\
$C_{8}$ & 0.4253785 & 0.42123807 \\
$C_{10}$ & 0.178100 & 0.17473318 \\
$B$ & 1.241314 & 1.4826 \\
\hline \hline
\end{tabular}

TABLE III. Parameters of the two versions of the Aziz ${ }^{4} \mathrm{He}^{-}{ }^{4} \mathrm{He}$ potential. 


\begin{tabular}{|c|c|c|}
\hline \multirow[b]{2}{*}{ Ref. } & \multicolumn{2}{|c|}{${ }^{4} \mathrm{He}-{ }^{4} \mathrm{He}$ binding energy $(\mathrm{mK})$} \\
\hline & HFDHE2 & HFD-B \\
\hline This work & 0.8301249029 & 1.6854110471 \\
\hline 13 & 0.8301 & 1.6854 \\
\hline 14 & 0.830 & - \\
\hline 15 & 0.829 & - \\
\hline 116 & 0.8299 & - \\
\hline
\end{tabular}

TABLE IV. Binding energies of ${ }^{4} \mathrm{He}_{2}$ di-atomic molecule for the two versions of the Aziz potential.

\begin{tabular}{|c|c|c|c|c|}
\hline \multirow[b]{2}{*}{$\ell$} & \multicolumn{2}{|c|}{ 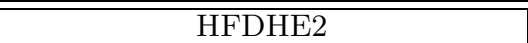 } & \multicolumn{2}{|c|}{$\overline{~ H F D-B ~}$} \\
\hline & $E(\mathrm{~K})$ & $\Gamma(\mathrm{K})$ & $E(\mathrm{~K})$ & $\Gamma(\mathrm{K})$ \\
\hline 1 & 0.334 & 1.822 & 0.339 & 1.795 \\
\hline 2 & 2.164 & 6.825 & 2.179 & 6.774 \\
\hline 4 & 11.954 & 27.196 & 12.005 & 27.000 \\
\hline 5 & 20.478 & 42.949 & 20.518 & 42.603 \\
\hline
\end{tabular}

TABLE V. Energies and widths of the lowest resonant states generated by the two versions of the Aziz potential in several partial waves.

\begin{tabular}{|c|c||r|r|}
\hline \hline \multicolumn{2}{|c|}{ HFDHE2 } & \multicolumn{2}{c||}{ HFD-B } \\
\hline$E(\mathrm{~K})$ & $\ell$ & $E(\mathrm{~K})$ & $\ell$ \\
\hline 0.334 & $0.525+i 0.429$ & 0.339 & $0.535+i 0.424$ \\
2.164 & $1.408+i 0.778$ & 2.179 & $1.416+i 0.773$ \\
5.930 & $2.294+i 1.065$ & 5.963 & $2.303+i 1.060$ \\
11.954 & $3.182+i 1.332$ & 12.005 & $3.191+i 1.326$ \\
20.478 & $4.070+i 1.590$ & 20.518 & $4.078+i 1.584$ \\
\hline \hline
\end{tabular}

TABLE VI. Regge poles corresponding to resonances generated by the two versions of the Aziz potential. 


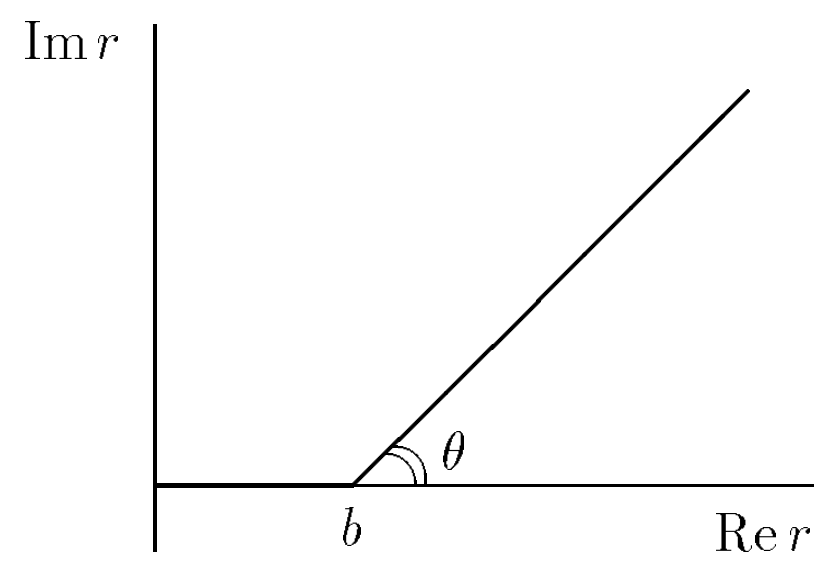

FIG. 1. Deformed contour for integration of the differential equations

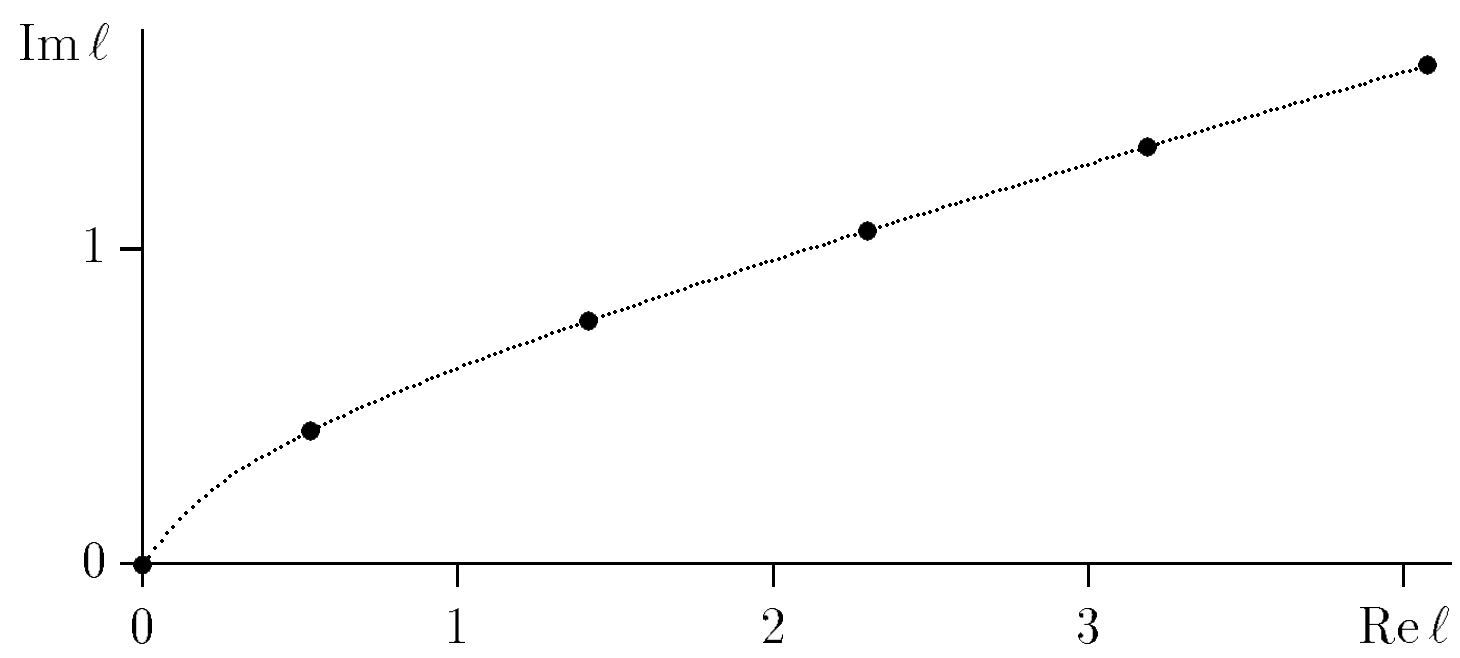

FIG. 2. Regge trajectory for the HFD-B potential. Filled circles indicate bound and resonant states. 